\title{
The Filter Design on Impedance control of Via
}

\author{
Xu Xiaofei ${ }^{12}$ \\ (1.School of Electronic and Information Engineering, Beijing Jiaotong University, Beijing, 100044; \\ 2.School of Automation, Beijing Information Science and Technology University, Beijing, 100192)
}

Keywords : SI; Via; Filter; Impedance control

\begin{abstract}
According to the specific environmental noise of the detection system, via holes was caused impedance discontinuities, parasite capacity and inductance which resulted in signal reflection and attenuation, and hence deterioration of signal integrity(SI). The new idea was put in the paper, the specific noise filter was designed on impedance control of via and interconnect. Capacitive coupling of via was considered, and electrical characteristics of hole model was simulated with different PCB plate, and the equivalent circuit model was added to effective parameters by HFSS and ADS collaborative simulation software; The power noise filter was designed and tested. The experiment data showed that the method can be extended to signal detection system with arbitrary single frequency noise interference in.
\end{abstract}

\section{Introduction}

In high-speed digital board designs, every slightest discontinuity on the board had to be considered carefully, especially via holes, which were abundantly used in high-speed multilayer PCB. As frequency increased and signal rise time reduced, via holes caused impedance discontinuity, parasite capacity and inductance which resulted in signal reflection and attenuation, and hence deterioration of signal integrity(SI). The paper carried out a comprehensive study of the impacts of various via parameter on impedance discontinuities of single ended via by vector network analyzer. The via parameters included diameter, pad diameter and anti-pad diameter. Furthermore, impedance continuities and SI for via holes were greatly improved by providing current path for signal in via. Based on the multilayered structure of PCB such as the hole, wire and conductor: First, the reflection coefficient and transmission coefficient values ${ }^{[1-5]}$ of hole model was built by HFSS, which we did simulation of high speed signal for its reflection coefficient and transmission coefficient values when crossed the hole, and computed the value of capacitance and inductance impedance caused by the distribution effect of the hole with the ADS collaborative simulation software. Then, we needed to set the parameter in the HFSS and ADS the physical structure and the theoretical model of the hole size ${ }^{[6-8]}$. In addition to combining the low frequency acceleration signal, detecting the transmission requirements of the actual signal, the design rules of the system PCB, testing the approximate circuit module, improving the noise ratio of input signal, including the characteristic of eliminating the coupling of via and interconnect, designing the power noise filter etc. Finally, above all it need to be done, and we had made signal integrity transmission come true ${ }^{[9-12]}$.

\section{System Introduction}

In this paper, the detection system could be simplified to a linear mathematical model, was showed in Fig.1. The author team had put forward solutions focused on development of via, because it can affect the signal transmission too much at low frequency. Considered from two aspects of cost and signal quality, to choose a reasonable size of the via, properly design the 
structure size and layout out the via, combine with the via adding a certain amount of series inductance capacitance impedance values to system to reduce the signal integrity and the effect of decoupling capacity, high-speed PCB design had a certain guiding significance. At the same time, covered with mental on fluctuation signal line of the circuit board, could reduce the signal transmission attenuation and electromagnetic interference of the circuit surface. The mathematical model of system information transmission process was shown in Fig.1:

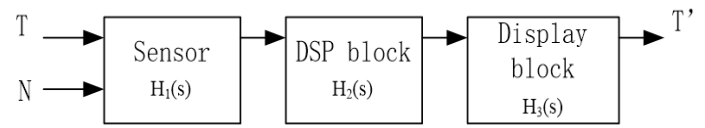

Fig.1 mathematical model of general detection system

The impedance control of via was coupled and designed in this paper building of notch filter signal processing module $\mathrm{H}_{2}(\mathrm{~s})$. The relationship between input signal and output signal of this system was simplified as Eq.1:

$$
T^{\prime}(s)=H_{1}(s) \cdot H_{2}(s) \cdot H_{3}(s) \cdot[T(s)+N(s)]
$$

As shown in Eq.1, the ultimate goal of this system was to keep the output signal T' follow the input signal $\mathrm{T}$ and remove noise signal $\mathrm{N}$.

\section{The equivalent impedance analysis of via}

Combination with HFSS software modeling simulation calculated parameters of via, using ADS circuit simulation software solve equivalent impedance of via model, designing specific lumped parameter notch filter to deal with signal integrity problem caused by power frequency signal noise in via and interconnect of test system.

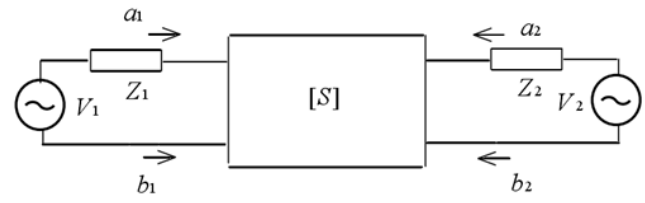

Fig.2 The impedance of via model

Where,Fig. 2 described a system which was stimulated by $\mathrm{V}_{1}$ and $\mathrm{V}_{2}, \mathrm{a}_{1}, \mathrm{a}_{2}, \mathrm{~b}_{1}, \mathrm{~b}_{2}$, respectively, represent the power of incident wave and reflected wave of input and output port. Assumed that, the system is linear, the scattering parameter (S) described the relationship between the two ports incident power and reflection power, rather than the relationship of voltage and current, application of S parameter measurement and calibration becomes easier, S parameter definitions was shown.

That via impedance matching was to make the load impedance and the source impedance conjugate matching, to gain the maximum power transmission of useful signal, and minimize the power loss on the feeder line. The method to realize the impedance matching is to insert a passive network between the source and load, the system was designed based on low frequency circuit, if designed with the method of micro strip line, its wavelength associates with the signal frequency, that would lead to the micro strip line too long, circuit board area too big, so using LC discrete component matching.

Equivalent circuit of the via physical model via electrical model can be equivalent to LC filter circuit, the equivalent LC value is shown in Eq. $2^{[4]}$ :

$$
C=1.41 \varepsilon_{r} T \frac{D_{1}}{D_{2}-D_{1}}
$$


Where, $\mathrm{C}$ for via parasitic capacitance, the unit was $\mathrm{F} ; \mathrm{L}$ for hole of parasitic inductance, the unit for $\mathrm{H} ; \mathrm{D}_{1}$ for hole diameter, the unit for the mil; $\mathrm{D}_{2}$ was the diameter of the power supply area, the unit for the mil; $\mathrm{T}$ was the thickness of the printed circuit board, the unit for the mil; Epsilon $\mathrm{r}$ for relative dielectric constant of substrate material, the unit for the $\mathrm{F} / \mathrm{m} ; \mathrm{H}$ was the height of the hole (equals the thickness plate), the unit for the mil; D for the inner diameter of the hole, the unit for the mil.

\section{The specific filter design on via}

Based on the collaborative method this paper used via model to design specific noise filter research flow chart shown as follow: First, based on $50(\mathrm{~Hz})$ notch filter index determine the Q value and $\mathrm{M}$ matrix(circuit curve); Then, based on software simulation and actual measurement calculate the equivalent capacitance coupling value of via and interconnect; Last, by using HFSS and ADS to build a field circuit model collaborative simulation, make sure via machining parameters and matching impedance circuit.

\section{Via physical model}

First, in the full-wave simulation software HFSS, simulation excitation spectrum signal acquire the scattering parameters of pore structure by measuring, set up three-dimensional physical model of via, its structure was a hole connecting two layer micro strip line, signal via size set should reference ${ }^{[6]}$ research conclusions: for FR4 PCB material, thickness of 50 (mil), dielectric constant $\square_{\mathrm{r}}$ is $2 \sim 4.4$, the line width is 27.5 (mil) change in length from 200 - 360 (mil), thickness of 0.6 (mil), the changes of via inner diameter from $5 \sim 10$ (mil), outer diameter change from $15 \sim 25$ (mil), as shown in Fig. 2 set micro strip lumped port $\mathrm{P}_{1}$ and $\mathrm{P}_{2}$.In the simulation, sweep signal frequency option $1 \sim 1 \mathrm{GHz}$ frequencies.
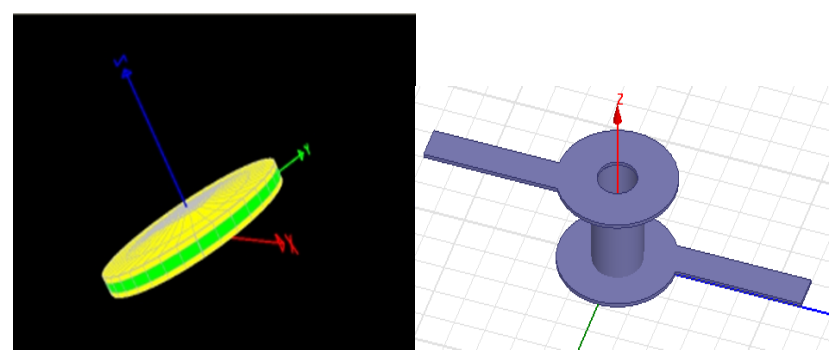

Fig.3 Hollow and solid via simulation modeling

Where, in Fig.3, through the electromagnetic simulation analysis of via design parameters, for reasons of engineering design and plate processing, 2(mil) in accuracy was allowed. Through HFSS software modeling and simulation, the rationality of the model and the validity of the design parameters were proved. When via $\mathrm{d}=10(\mathrm{mil}), \mathrm{D} 2=25(\mathrm{mil}), \mathrm{h} 2=10(\mathrm{mil})$ aperture changes between $0.2 \sim 0.6(\mathrm{~mm}), \mathrm{C}_{\mathrm{k}}$ values of via capacitance changed little at low frequencies, about $40 \mathrm{fF}$.

\section{The filter design on impedance matching circuit}

Import the S parameter calculated from the full field simulation of HFSS to the ADS software, establish the equivalent circuit model, as shown in Fig.4, LC second-order filter take 25(Hz)-0(db), $50(\mathrm{~Hz})-12(\mathrm{db})$, the equivalent capacitance $\mathrm{C}_{0}$ equivalent to piezoelectric sensor capacitance $\mathrm{Cs}$ parallel via $C_{k}$ values, at low pass circuit $25(\mathrm{~Hz})$, intrinsic impedance $50 \Omega$ and normalization method to calculate the inductance $\mathrm{L}$ around $300 \mathrm{mH}$, based on this to series and parallel connect capacitor and inductor to optimization design. 


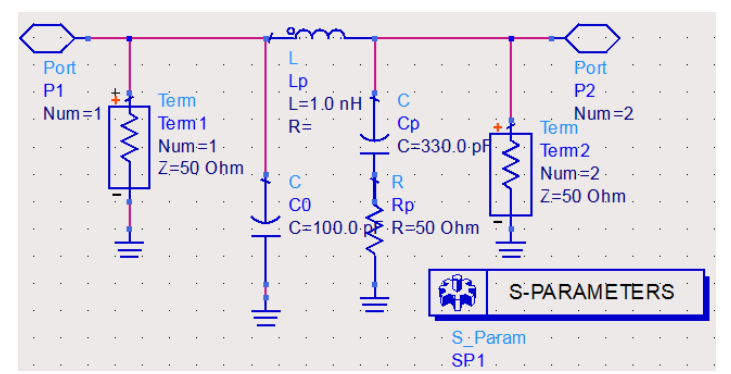

Fig.4 circuit model simulation of ADS software

By checking Smith chart, we could learn from impedance value of minimum $S_{11}$, the complete system matching parameter, as shown in Fig.5.

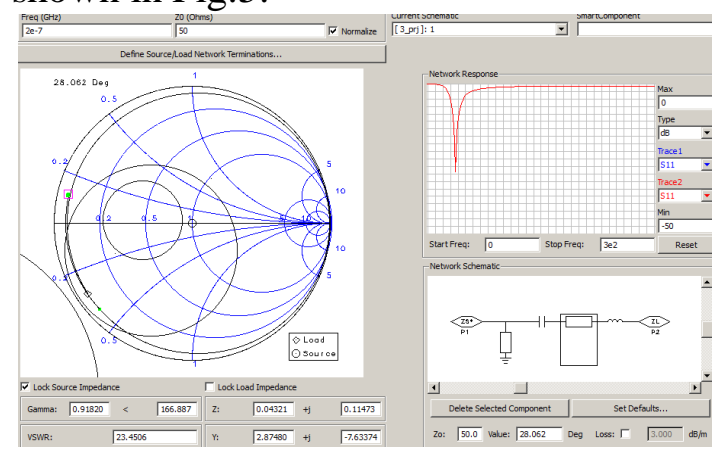

Fig.5 Smith chart impedance matching

By using the notch filter apply signal generator to input $1000 \mathrm{mV}$, frequency variation of incentive sine signal to observe scattering parameters. Through the simulation data and considering the actual circuit design error, chose the notch filter circuit design parameter as follow: equivalent capacitance $\mathrm{C}_{\mathrm{p}}=0.33(\mathrm{uF}), \mathrm{R}_{1}=11(\mathrm{k} \Omega), \mathrm{R}_{2}=50(\mathrm{k} \Omega)$, the depth of the notch was $-13.98(\mathrm{~dB})$, notch width was $25(\mathrm{~Hz})$.

\section{Conclusions}

The method of the paper could be applied to an arbitrary single frequency noise interference signal detection system; As shown in Fig.6, the 50(Hz) power frequency noise signal was filtered by "via".
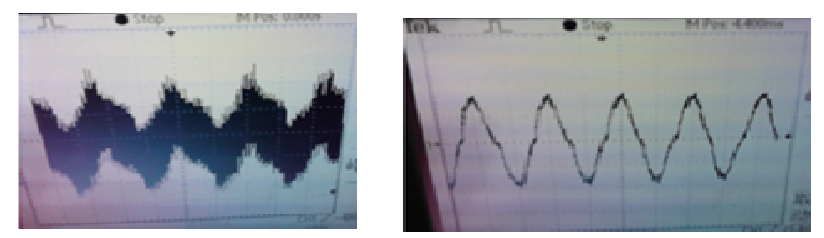

Fig. 6 The input and output signal of via filter

The impedance control of via was achieved by collaborative simulation to find the exact position of the resonance and the optimal value of circuit parameters. And the specific bandwidth filter was designed by choosing the appropriate via. Via design should be considered respectively according to different routing structures including coaxial line and micro strip line, strip line and so forth. At the same time, via equivalent electrical model, or extraction of the equivalent parameters was required to further study.

\section{ACKNOWLEDGMENTS}

This work was financially supported by Beijing science foundation of the state education commission(KM201411232006), Beijing Natural Science Foundation (4122028), the Funding 
Project for Academic Human Resources Development in Institutions of Higher Learning under the Jurisdiction of Beijing Municipality (PHR201107218) and the Beijing Postgraduate Joint Training Base Project (71C1311016,71D1311007).

\section{Reference}

[1] Kumar, T.R.S , Venkatesh, C. Shielding Effectiveness comparison of rectangular and cylindrical enclosures with rectangular and circular apertures using TLM modeling[C]. //Applied Electromagnetics Conference. 2009. 1-4.

[2] Liuping Wang, Yougang Gao, Yuanmao Shen, Fangming Ruan. Analysis on Shielding Effectiveness of Aperture Arrays with Different Spacing[C]. //International Symposium on Electromagnetic Compatibility. 2007. 333-336.

[3] Doncov, N., Wlodarczyk, A.J., Scaramuzza, R., Trenkic, V.. Compact TLM model for air-vents[J]. Electronics Letters. 2002. 38. 887-889.

[4] N. Fichtner, P. Russer. An accelerated hybrid TLM-IE method for the investigation of shielding effectiveness[J]. Advances in Radio Science. 2010. 13-18.

[5] Silveira, J.L., Benhassine, S., Pichon, L., Raizer, A.. Analysis of the shielding effectiveness of a rectangular enclosure with apertures by TLM-TD[C]. //The Fourth International Conference on Computation in Electromagnetics. 2002.

[6] Christopoulos, C. . Application of the TLM method to equipment shielding problems[C]. //IEEE International Symposium on Electromagnetic Compatibility. 1998. 1. 188-193.

[7] Argus, P., Fischer, P., Konrad, A., Schwab, A.J.. Efficient modeling of apertures in thin conducting screens by the TLM method[C]. //IEEE International Symposium on Electromagnetic Compatibility. 2000. 1. 101-106.

[8] Min Li, Joe Nuebel, James L. Drewniak. EMI from Airflow Aperture Arrays in Shielding Enclosure-Experments, FDTD, and MOM Modeling[J]. IEEE Transactions on Electromagnetic Compatibility. 2000. 42(3). 265-275.

[9] Chao Feng, Zhongxiang Shen. A hybrid FD-MoM technique for predicting shielding effectiveness of metallic enclosures with apertures[J]. IEEE Transactions on Electromagnetic Compatibility. 2005. 47. 456-462.

[10] Cai X W, Tian Y, Tong L. Electromagnetic characterization analysis of the connecting structure of

the via in multilayered microwave circuit[J]. Global Symposium Millimeter Waves, Nanjing (China), April

2008: 21-24.

[11] Kohlmeier D. Via modeling[J]. Printed Circuit Design \& Manufacture. 2005, 22(12):26-28.

[12] Richard W Y C, See K Y, Chua E K. Comprehensive analysis of the impact of via design on high-speed signal

integrity[C]. 9th Electronics Packaging Technology Conference, Cambridge(USA), 2007: 262-266. 